\title{
Optimal Control for Allen-Cahn Equations Enhanced by Model Predictive Control
}

\author{
Peter Benner* Martin Stoll ** \\ * Max Planck Institute for Dynamics of Complex Technical Systems, \\ Magdeburg, Germany (e-mail: benner@mpi-magdeburg.mpg.de). \\ ** Max Planck Institute for Dynamics of Complex Technical Systems, \\ Magdeburg, Germany (e-mail: stollm@mpi-magdeburg.mpg.de)
}

\begin{abstract}
The Allen-Cahn equation is a simple model of a nonlinear reaction-diffusion process. It is often used to model interface motion in time, e.g. phase separation in alloys. It has applications in many areas including material sciences, biology, geology, as well as image processing. We will consider a simple scalar Allen-Cahn equation subject to distributed control. Here, the nonlinear reaction term is obtained from using the standard double-well potential, leading to a cubic nonlinearity. We will describe a nonlinear feedback control strategy based on the concept of Model Predictive Control (MPC). We also show how to obtain the open-loop trajectory and control using numerical techniques for PDE-constrained optimization. The feedback control scheme is then applied to the spatially semi-discretized nonlinear optimal control problem. For the prediction and control step within the MPC scheme, we apply a linear-quadratic regulator/Gaussian design problem. The arising computational challenge consisting in solving the associated large-scale algebraic Riccati equations has already been shown in the literature to be feasible using reasonably fine discretizations.
\end{abstract}

Keywords: Allen-Cahn equation, model-based control, model-predictive control, LQG control, Riccati equations, numerical algorithms

\section{INTRODUCTION}

The Allen-Cahn equation was introduced in Allen and Cahn (1979) to model the motion of antiphase boundaries in crystalline solids. It has since found widespread application in imaging (Kay and Tomasi (2007); Sarbu (2010)), material science (Deckelnick et al. (2005); Allen and Cahn (1979)), and biology (Malvasi et al. (2011)).

In the following we consider the minimization of an objective function given by

$$
J(y, u)=\frac{1}{2} \int_{Q_{\mathrm{ref}}}\left(y-y_{\mathrm{ref}}\right)^{2}+\frac{\beta}{2} \int_{Q} u^{2}
$$

where $Q=[0, T] \times \Omega$ is a space-time cylinder with $\Omega$ a two or three-dimensional domain of sufficient regularity. Here $y$ is the state, $y_{\text {ref }}$ is the desired reference state, and $Q_{\mathrm{ref}}=[0, T] \times \Omega_{\mathrm{ref}}$ with $\Omega_{\mathrm{ref}} \subset \Omega$ is the observation space. The control $u$ is linked to the state via the controlled AllenCahn equation

$$
\varepsilon y_{t}=\varepsilon \triangle y-\varepsilon^{-1} \Psi^{\prime}(y)+u
$$

with $\varepsilon>0$. The boundary conditions for this problem are of Neumann type, i.e., $\frac{\partial y}{\partial n}=0$, and the initial condition is given by $y(0, x)=y^{0}$. The potential function $\Psi$ plays a crucial role and for the sake of simplifying the development of a nonlinear feedback control concept, we choose the wellknown double well potential defined by $\Psi(y)=\frac{1}{4}\left(1-y^{2}\right)^{2}$. Other nonsmooth potential functions that may have better properties regarding the modeling of physical properties will be discussed in future work.
For the existence of solutions to the problems similar to the one given above we refer the interested reader to Farshbaf-Shaker (2011); Colli and Sprekels (2012). We simply assume here that all derivatives are well defined and the necessary functions are sufficiently smooth.

Our approach to solve the above given optimization problem follows the optimize-then-discretize procedure (see Hinze et al. (2009) for an introduction to this field). For this, we state in Section 2 the nonlinear, infinitedimensional first order conditions and solve these using a Newton technique. The corresponding Newton system is then discretized using a finite element discretization, which in turn results in the solution of a large, sparse, and structured linear system that needs to be solved at every step of the Newton scheme. In order to correct for unwanted disturbances and modeling errors in a practical application of the optimized control, it is necessary to have a feedback mechanism to compensate for deviations from the optimized trajectory. For this, we suggest a Model Predictive Control (MPC) scheme (for which we assume the reader to be familiar with the basic concept) based on a Linear-Quadratic Gaussian (LGQ) design as suggested for general nonlinear reaction-diffusion systems in Benner and Hein (2013). This approach will be reviewed in Section 3. Numerical results for the whole design process including the feedback control will be reported in a future extended version of this discussion paper; first results obtained with the open-loop control computed using the optimization 
procedure from Section 2 are contained in Section 4. We discuss our findings and open problems in Section 5.

\section{OPTIMIZATION OF THE ALLEN-CAHN SYSTEM}

For solving the optimization problem related to (1)-(2), we follow the formal Lagrange approach (see Tröltzsch (2010); Ito and Kunisch (2008)) and form the Lagrangian

$$
\begin{aligned}
\mathcal{L}(y, u, p)= & J(y, u)+ \\
& \int_{Q} p\left(y_{t}-\Delta y-\varepsilon^{-2} y\left(y^{2}-1\right)-\varepsilon^{-1} u\right),
\end{aligned}
$$

employing the homogeneous Neumann boundary conditions and considering the simplified situation $\Omega_{\text {ref }}=\Omega$. The first order conditions for this problem can be obtained using the Fréchet derivative of $\mathcal{L}$. We thus obtain

$$
\begin{aligned}
& \mathcal{L}_{y}=\left(y-y_{\mathrm{ref}}\right)-p_{t}-\Delta p-\frac{1}{\varepsilon^{2}}\left(3 y^{2}-1\right) p=0, \\
& \mathcal{L}_{u}=\beta u+\frac{1}{\varepsilon} p=0 \\
& \mathcal{L}_{p}=y_{t}-\triangle y-\varepsilon^{-2} y\left(y^{2}-1\right)+\varepsilon^{-1} u=0 .
\end{aligned}
$$

A Lagrange-Newton or SQP (sequential quadratic programming) approach (see, e.g., Nocedal and Wright (2006)) now solves these nonlinear equations using a Newton scheme. We formalize this by writing (4) to (6) as $G(x)=0$, which we approximately solve using an iteration based on

$$
G^{\prime}(\bar{x}) s=-G(\bar{x})
$$

where $\bar{x}$ is the previous iterate in the Newton step and $s=x-\bar{x}$ represents the difference between the new and previous approximation to the solution. The differentiation of (4) to (6) results in the following Newton system

$$
\underbrace{\left[\begin{array}{ccc}
s_{y}-\frac{1}{\varepsilon^{2}}(6 \bar{y}) \bar{p} s_{y} & 0 & -\left(s_{p}\right)_{t}-\Delta s_{p}-\frac{1}{\varepsilon^{2}}\left(3 \bar{y}^{2}-1\right) \\
0 & \beta s_{u} & \frac{1}{\varepsilon} s_{p} \\
\left(s_{y}\right)_{t}-\triangle s_{p}-\frac{1}{\varepsilon^{2}}\left(3 \bar{y}^{2}-1\right) & \frac{1}{\varepsilon} s_{u} & 0
\end{array}\right]}_{=: G^{\prime}(\bar{x}) s .}
$$

Note that this can be formulated such that we solve for the updated approximation $x=\left[y^{T}, u^{T}, p^{T}\right]^{T}$ rather than the step. This is done by considering the structure of the right hand side $-G(\bar{x})$ and the explicit computation of the new right hand side $G^{\prime}(\bar{x}) \bar{x}-G(\bar{x})$. At this stage we discretize (7) using finite elements in space and an implicit Euler scheme in time. Performing an all-at-once approach, i.e., we solve for all time-steps simultaneously, we obtain a linear system of the following form:

$$
\underbrace{\left[\begin{array}{ccc}
\tau \mathcal{M}_{y} & 0 & -\mathcal{K}^{T} \\
0 & \beta \tau \mathcal{M}_{u} & \varepsilon^{-1} \tau \mathcal{N} \\
-\mathcal{K}^{T} & \varepsilon^{-1} \tau \mathcal{N} & 0
\end{array}\right]}_{=: \mathcal{A}}\left[\begin{array}{l}
\mathbf{y} \\
\mathbf{u} \\
\boldsymbol{p}
\end{array}\right]=b .
$$

Here $\mathcal{K}$ represents a one-shot discretization of the PDEconstraint, i.e.,

$$
\mathcal{K}=\left[\begin{array}{cccc}
L_{0} & & & \\
-M & L_{1} & & \\
& \ddots & \ddots & \\
& & -M & L_{N_{t}}
\end{array}\right]
$$

where $L_{i}=M+\tau K-M^{\frac{1}{\varepsilon^{2}}\left(3 \bar{y}\left(t_{i}\right)^{2}-1\right)}$. Here $M$ and $K$ are the mass and stiffness matrix, respectively. The matrix $M^{f}$ is a mass-like matrix whose entries are obtained when the test functions are integrated against the function $f$. Furthermore, $\mathcal{M}_{y}=\operatorname{blkdiag}\left(M^{1-\frac{1}{\varepsilon^{2}}\left(6 \bar{y}\left(t_{i}\right)\right) \bar{p}\left(t_{i}\right)}\right) \forall i$ and $\mathcal{M}_{u}=\mathcal{N}=\operatorname{blkdiag}(M, \ldots, M)$. Our discretization here follows an approach presented in Hinze et al. (2008); Stoll and Wathen (2013), which follows the paradigm that the discretize-then-optimize and the optimize-then-discretize approach coincide. More details on the derivation of allat-once methods can be found in Stoll and Wathen (2010).

As we now need to solve the linear system (8) at every step of the Newton iteration, the iterative scheme to carry out the approximation of the solution has to be efficient. Due to the size of $\mathcal{A}$, the use of direct solvers (Duff et al. (1989)) is out of the question. We therefore employ a Krylov subspace method, namely the bi-conjugate gradient method (BICG) introduced in Fletcher (1976). Other methods (see, e.g., Saad (2003)) are of course possible. Without additional acceleration by a preconditioner $\mathcal{P}$, the Krylov method typically converges very slowly. We now have to design the preconditioner $\mathcal{P}$ in such a way that it is cheap to apply but still resembles the matrix $\mathcal{A}$ as much as possible. Note that the all-at-once approach combined with a preconditioning technique is performed in a matrixfree way as we never form the matrix $\mathcal{A}$ explicitly and only use its multiplication with a vector. The choice of a nonsymmetric solver is due to the fact that the preconditioner $\mathcal{P}$ can become indefinite, which is not possible to use within symmetric solvers such as CG or MINRES.

Motivated by a result proposed by Murphy et al. (2000) we want to approximate the $(1,1)$ block

$$
A=\left[\begin{array}{cc}
\tau \mathcal{M}_{y} & 0 \\
0 & \beta \tau \mathcal{M}_{u}
\end{array}\right]
$$

and the Schur-complement

$$
S=\tau^{-1} \mathcal{K} \mathcal{M}_{y}^{-1} \mathcal{K}^{T}+\frac{\tau}{\beta} \mathcal{N} \mathcal{M}_{u}^{-1} \mathcal{N}^{T}
$$

well, as a good clustering of the eigenvalues typically leads to rapid convergence of the iterative solver. The block $A$ is easily approximated as we are dealing with lumped mass matrices obtained by using the trapezoidal rule with Q1 elements within the finite element discretization. We can therefore simply invert the matrix $A$ in our preconditioner. The construction of efficient Schur-complement approximations is typically more complicated and much of the research on preconditioners for PDE-constrained optimization has focused on this topic; see Pearson and Wathen (2012); Pearson et al. (2012); Kollmann and Zulehner (2012).

As a first step, a preconditioner ignoring the second term in $S$ has proven to be effective for related problems (Rees et al. (2010)) when rather large values of the regularization parameter $\beta$ are used, i.e., $\hat{S}_{1}=\tau^{-1} \mathcal{K} \mathcal{M}_{y}^{-1} \mathcal{K}^{T}$. We discuss this preconditioner here as its successors improving the parameter robustness typically are of a similar form. During the iterative process the action of $\hat{S}_{1}^{-1}=\tau^{-1} \mathcal{K}^{-T} \mathcal{M}_{y} \mathcal{K}^{-1}$ would, due to the block-triangular structure of $\mathcal{K}$, require the solution of the diagonal blocks of $\mathcal{K}$ representing the discrete Allen-Cahn block. As this is already not feasible for small mesh-sizes, we use a multigrid scheme of algebraic 
type to approximate the inverse of the diagonal blocks of $\mathcal{K}$. This means that only approximate solves with a discretized PDE are needed in the process of obtaining the solution to the control problem. Extensions of this preconditioner to improve on the robustness with respect to the crucial parameters are often of the form $\hat{S}_{2}=\tau^{-1}(\mathcal{K}+$ $\hat{\mathcal{M}}) \mathcal{M}_{y}^{-1}(\mathcal{K}+\hat{\mathcal{M}})^{T}$, where $\hat{\mathcal{M}}$ represents a block-diagonal perturbation of the discretize time-dependent PDE that is designed in such a way to also match the previously ignored second term in $S$ (see Pearson et al. (2012)).

\section{A MODEL PREDICTIVE CONTROL APPROACH}

For applying an efficient feedback control strategy, we assume that the necessary control force can be applied in a subdomain $\Omega_{u}$ of $\Omega$. The control system for deriving the feedback controller will be derived for the deviation from the optimized state $y_{\text {opt }}$ and control $u_{\text {opt }}$ computed using the approach in the previous section. Note that $y_{\text {opt }}$ may deviate from $y_{\text {ref }}$, but the controller derived here is meant to keep the system as close as possible to the optimized state only. If the optimization leads to a trajectory too far from the desired state, it is unlikely that one can get closer with a feedback control strategy. So the quality of the eventual trajectory is basically determined by the open-loop control/optimization, and the feedback controller presented here is meant to ensure that this optimized trajectory is then realized in praxis.

Assuming that controlling the magnitude of the control signal suffices, we use the separation of variables approach for $u(x, t)=\tilde{b}(x) v(t)$. Now using a spatial semidiscretization based on the same finite element mesh used for the optimization scheme derived in the previous section, we obtain the following finite-dimensional optimal control problem, using $\mathbf{y}, \mathbf{y}_{\text {opt }}$, and $\mathbf{u}$ to denote the resulting vector quantities (e.g., for linear nodal elements, the state and control approximations in the nodes of the finite element mesh):

$$
\begin{aligned}
J(\mathbf{y}, \mathbf{u})=\frac{1}{2} \int_{0}^{T}\left(\mathbf{y}(t)-\mathbf{y}_{\mathrm{opt}}(t)\right)^{T} M_{\mathrm{ref}}\left(\mathbf{y}(t)-\mathbf{y}_{\mathrm{opt}}(t)\right) d t \\
+\frac{\beta}{2} \int_{0}^{T} v(t)^{T} v(t) d t \\
+\frac{1}{2}\left(\mathbf{y}(T)-\mathbf{y}_{\mathrm{opt}}(T)\right)^{T} R\left(\mathbf{y}(T)-\mathbf{y}_{\mathrm{opt}}(T)\right)
\end{aligned}
$$

subject to

$$
M \dot{\mathbf{y}}(t)=S \mathbf{y}-\varepsilon^{-2} F(\mathbf{y})+\varepsilon^{-1} B \mathbf{v}
$$

where $M$ and $S$ represent the mass and negative stiffness matrix, respectively, obtained from the spatial discretization of (2). The control operator matrix $B$ is obtained by the FEM ansatz when testing against $b, M_{\text {ref }}$ is the mass matrices obtained by the FEM discretization on the observation domain $\Omega_{\text {ref }}$, and the penalization term for the deviation from the end state $\mathbf{y}_{\text {opt }}(T)$ involving a positive definite $R$ is introduced to guarantee stabilization properties of the MPC approach (Ito and Kunisch (2001)).

In order to model the unknown disturbances, we add noise terms to the state equation $\left(F w_{s}(t)\right)$, the observed state $\left(w_{\text {ref }}\right)$, and the initial conditions on each new time horizon in our MPC scheme $\left(w_{0}\right)$, all assumed to be independent Gaussian white noise processes with covariance matrices
$W_{s}, W_{\text {ref }}, W_{0}$, respectively. Our MPC ansatz then consists in solving in each prediction step a linear-quadratic control problem using a Gaussian design, leading to a (local) LQG controller. The scheme, based on a given time grid

$$
0=t_{0}<t_{1}<\ldots<T_{N}=T,
$$

and user-defined prediction and control horizons $T_{p}$ and $T_{c} \ll T_{p}$ is then as follows:

(1) Prediction and optimization step on $\left[t_{i}, t_{i}+T_{p}\right]$ : linearize (10) around the optimized trajectory $\mathbf{y}_{\text {opt }}$ to obtain $S_{F}=F^{\prime}\left(\mathbf{y}\left(t_{i}\right)\right)$. Defining the deviation from the optimal trajectory and control as $\mathbf{z}(t)=\mathbf{y}(t)-$ $\mathbf{y}_{\text {opt }}(t), \tilde{u}(t):=v(t)-v_{\text {opt }}(t)$ (with $v_{\text {opt }}(t)$ obtained from the separation of variables approach applied to $\left.u_{\text {opt }}(x, t)\right), A:=S-\varepsilon^{-2} S_{F}$, we obtain the linear state equation

$$
M \dot{z}(t)=A z(t)+\varepsilon^{-1} B \tilde{u}(t)+F w_{s}(t)
$$

Denoting the discrete observation operator i.e. the restriction to $\Omega_{\text {ref }}$, by $C$, we solve the algebraic Riccati equation (ARE)

$$
\begin{gathered}
0=M^{T} X A+A^{T} X M+C^{T} M_{\mathrm{ref}} C \\
-\frac{1}{\beta \varepsilon^{2}} M^{T} X B B^{T} X M
\end{gathered}
$$

for its unique symmetric positive semidefinite solution $X_{\star}$ and compute the feedback (gain) matrix

$$
K=-\frac{1}{\beta \varepsilon} B^{T} X_{\star} M
$$

Solve the filter ARE (FARE)

$$
\begin{aligned}
0= & A \Sigma M^{T}+M \Sigma A^{T}+F W_{s} F^{T} \\
& -M \Sigma C^{T} W_{\text {ref }}^{-1} C \Sigma M^{T}
\end{aligned}
$$

for its unique symmetric positive semidefinite solution $\Sigma_{\star}$ and compute the estimator gain

$$
L=\Sigma_{\star} C^{T} W_{\mathrm{ref}}^{-1}
$$

(2) Implementation step on $\left[t_{i}, t_{i}+T_{c}\right]$ : Feed the real system on $\left[t_{i}, t_{i}+T_{c}\right]$ with

$$
v(t)=v_{\text {opt }}(t)-K\left(\hat{\mathbf{y}}(t)-\mathbf{y}_{\text {opt }}(t)\right)
$$

where the state estimation $\hat{\mathbf{y}}(t)$ is obtained by first solving the nonlinear system (10) with initial condition $\mathbf{y}\left(t_{i}\right)+w_{0}\left(t_{i}\right)$ on $\left[t_{i}, t_{i}+T_{c}\right]$ to obtain $\mathbf{y}(t)$ and then the estimator equation

$$
\dot{\hat{\mathbf{z}}}(t)=(A-L C) \hat{\mathbf{z}}(t)+B \tilde{u}(t)+L C \mathbf{y}(t)
$$

for $\hat{\mathbf{z}}(t)=\hat{\mathbf{y}}(t)-\mathbf{y}_{\mathrm{opt}}(t)$.

(3) Receding Horizon Step:

Update $t_{i+1}=t_{i}+T_{c}$ and go to the first step.

Under some technical assumptions, the above scheme ensures $\mathbf{z}(t) \rightarrow 0$ for $T \rightarrow \infty$ and thus stabilization of the disturbed state to the desired optimal reference state; see Ito and Kunisch (2001, 2006); Benner and Hein (2010). For further details of the implementation of this approach, we refer to Benner and Hein (2010) and an upcoming extended version of this note.

\section{NUMERICAL EXAMPLE}

Here, we present the numerical results obtained by the open-loop (optimization) procedure described in Section 2 for a model problem. Full numerical tests using the MPC 


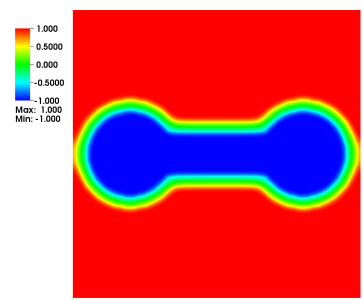

(a) Initial observed state

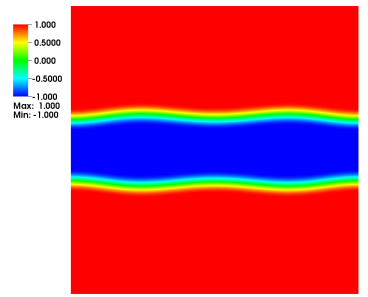

(b) Reference state after 50 timesteps

Figure 1: Initial reference state and reference state at timestep 50 obtained by evaluating the Allen-Cahn equation with volume constraints and a double obstacle potential.
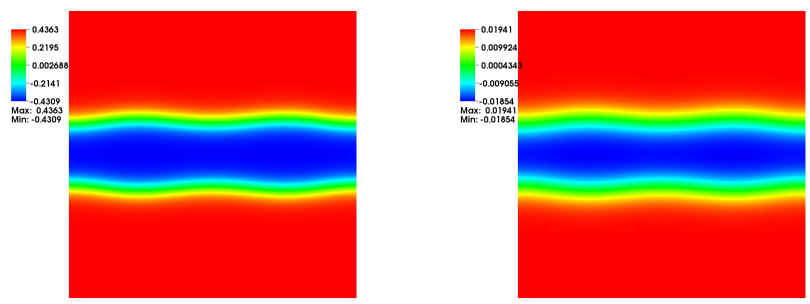

(a) Computed Control after 50 time-steps

(b) Computed state after 50 time-steps

Figure 2: Computed control and state at time-step 50 obtained via the Lagrange-Newton method.

approach for compensating deviations from the optimized trajectory will be provided elsewhere.

The desired state $\mathbf{y}_{\text {ref }}$ is obtained from employing the Allen-Cahn equation with volume constraints and a nonsmooth obstacle potential to a two-dimensional dumbbell (shown in Figure 1b). We used $\varepsilon=0.05$ and time steps of size $\triangle_{T}=0.001$. For details on the Allen-Cahn equation with volume constraints we refer to Blank et al. (2011); Garcke et al. (2008); Taylor and Cahn (1994).

We show the solution obtained for a rather large regularization parameter $\beta=10^{-1}$ in Figure 2. Table 1 shows the number of SQP iterations as well as the number of BICG iterations per SQP step. For the linear systems we used a stopping tolerance of $10^{-4}$ for the relative residual and the SQP method was stopped when the relative change between two consecutive solutions was less than $10^{-4}$. The control problem was solved for $\tau=0.01$ over the time interval $[0,1]$.
Table 1: Results for the solution of the control problem showing the number of BICG iterations for each SQP step.

\begin{tabular}{ccc}
\hline DoF & SQP & BICG \\
\hline & & $\beta=10^{-1}$ \\
\hline 5042223 & step 1 & 15 \\
& step 2 & 18 \\
& step 3 & 20 \\
& step 4 & 20 \\
& step 5 & 20 \\
& step 6 & 20 \\
\hline
\end{tabular}

\section{CONCLUSIONS}

We have investigated a model predictive control concept for Allen-Cahn equations. We have presented a numerical scheme to obtain an open-loop control based on a full discretization of the formal first-order necessary optimality system for the associated abstract optimal control problem. In order to compensate for deviations from the optimized trajectory when applying the optimal control in a practical situation, we suggest to use an MPC scheme based on an LQG design. This requires solving coupled algebraic Riccati equations which nowadays can be done efficiently for medium-grain discretizations. Future work will include the usage of coarse grids and model reduction to speed-up the MPC step with the aim of facilitating real-time computations. Another research direction will be to investigate vector Allen-Cahn equations as well as the study of different potential functions and problems with degenerate mobility term, leading to the Cahn-Hilliard system.

\section{REFERENCES}

S.M. Allen and J.W. Cahn. A microscopic theory for antiphase boundary motion and its application to antiphase domain coarsening. Acta Metallurgica, 27(6): 1085-1095, 1979.

W. Bangerth, R. Hartmann, and G. Kanschat. deal.IIa general-purpose object-oriented finite element library. ACM Trans. Math. Software, 33(4):Art. 24, 27 pages, 2007.

P. Benner and S. Hein. MPC/LQG for infinitedimensional systems using time-invariant linearizations. in D. Hömberg, F. Tröltzsch (eds.), System Modeling and Optimization. 25th IFIP TC 7 Conference, Berlin, Germany, September 12-16, 2011, pages 217-224, IFIP AICT, Vol. 391, Springer-Verlag, Heidelberg, 2013.

P. Benner and S. Hein. Model predictive control based on an LQG design for time-varying linearizations. Chemnitz Scientific Computing Preprints 0907, TU Chemnitz, March 2010. Available online at http://nbn-resolving.de/urn:nbn:de:bsz:ch1-201000221.

L. Blank, H. Garcke, L. Sarbu, and V. Styles. Primal-dual active set methods for Allen-Cahn variational inequalities with non-local constraints. Numerical Methods for Partial Differential Equations, 2011.

P. Colli and J. Sprekels. Optimal control of an allencahn equation with singular potentials and dynamic 
boundary condition. arXiv preprint arXiv:1212.2359, 2012.

K. Deckelnick, G. Dziuk, and C. M. Elliott. Computation of geometric partial differential equations and mean curvature flow. Acta Numerica, 14:139-232, 2005. doi: 10.1017/S0962492904000224.

I. S. Duff, A. M. Erisman, and J. K. Reid. Direct methods for sparse matrices. Monographs on Numerical Analysis. The Clarendon Press Oxford University Press, New York, 1989.

H. Farshbaf-Shaker. A penalty approach to optimal control of Allen-Cahn variational inequalities: MPECview. Technical report, University of Regensburg, 2011.

R. Fletcher. Conjugate gradient methods for indefinite systems. In Numerical analysis (Proc 6th Biennial Dundee Conf., Univ. Dundee, Dundee, 1975), pages 7389. Lecture Notes in Math., Vol. 506. Springer, Berlin, 1976.

H. Garcke, B. Nestler, B. Stinner, and F. Wendler. AllenCahn systems with volume constraints. Math. Models Methods Appl. Sci., 18(8):1347-1381, 2008.

M. Hinze, R. Pinnau, M. Ulbrich, and S. Ulbrich. $O p$ timization with PDE Constraints. Mathematical Modelling: Theory and Applications. Springer-Verlag, New York, 2009.

M. Hinze, M. Köster, and S. Turek. A Hierarchical Space-Time Solver for Distributed Control of the Stokes Equation. DFG Priority Program SPP1253-16-01, 2008. Available from http://www.am. uni-erlangen.de/home/spp1253/.

K. Ito and K. Kunisch. On asymptotic properties of receding horizon optimal control. SIAM J. Cont. Optim. 40:1455-1472, 2001.

$\mathrm{K}$. Ito and K. Kunisch. Receding horizon control with incomplete observations. SIAM J. Cont. Optim. 45(1):207-225, 2006.

K. Ito and K. Kunisch. Lagrange multiplier approach to variational problems and applications, volume 15 of Advances in Design and Control. Society for Industrial and Applied Mathematics (SIAM), Philadelphia, PA, 2008.

D.A. Kay and A. Tomasi. Colour image segmentation by the vector-valued Allen-Cahn phase-field model: a multigrid solution. IEEE Trans. Image Proc., 18:23302339, 2009.

M. Kollmann and W. Zulehner. A Robust Preconditioner for Distributed Optimal Control for Stokes Flow with Control Constraints. Numerical Mathematics and Advanced Applications 2011, 2013. doi: http://dx.doi.org/10.1007/978-3-642-33134-3_81.

A. Malvasi, A. Tinelli, S. Rahimi, G. D'Agnese, C. Rotoni, D. Dell'Edera, D.A. Tsin, and C. Cavallotti. A three-dimensional morphological reconstruction of uterine leiomyoma pseudocapsule vasculature by the allencahn mathematical model. Biomedicine \& Pharmacotherapy, 65(5):359-363, 2011.

M. F. Murphy, G. H. Golub, and A. J. Wathen. A note on preconditioning for indefinite linear systems. SIAM J. Sci. Comput, 21(6):1969-1972, 2000.

J. Nocedal and S. J. Wright. Numerical optimization. Springer Series in Operations Research and Financial Engineering. Springer, New York, second edition, 2006.
J. W. Pearson and A. J. Wathen. A new approximation of the schur complement in preconditioners for pde-constrained optimization. Numerical Linear Algebra with Applications, 19:816-829, 2012. doi: $10.1002 /$ nla. 814 .

J. W. Pearson, M. Stoll, and A. J. Wathen. Regularizationrobust preconditioners for time-dependent PDEconstrained optimization problems. SIAM J. Matrix Anal. Appl, 33(4):1126-1152, 2012.

T. Rees, H. Sue Dollar, and A. J. Wathen. Optimal solvers for PDE-constrained optimization. SIAM J. Sci. Comp., 32(1):271-298, 2010. doi: http://dx.doi.org/10.1137/080727154.

Y. Saad. Iterative methods for sparse linear systems. Society for Industrial and Applied Mathematics, Philadelphia, PA, 2003.

L. Sarbu. Primal-dual active set methods for AllenCahn variational inequalities. $\mathrm{PhD}$ thesis, University of Sussex, UK, 2010.

M. Stoll and A. Wathen. All-at-once solution of time-dependent PDE-constrained optimization problems. Technical Report, 2010.

M. Stoll and A. Wathen. All-at-once solution of timedependent Stokes control. J. Comp. Phys., 232:498-515, 2013.

J. E. Taylor and J. W. Cahn. Linking anisotropic sharp and diffuse surface motion laws via gradient flows. $J$. Statist. Phys., 77(1-2):183-197, 1994.

F. Tröltzsch. Optimal Control of Partial Differential Equations: Theory, Methods and Applications. American Mathematical Society, 2010. 